\title{
An Electromagnetic-Neurophysiological Model of Memory
}

\author{
Kanad Ray \\ Institute of Engineering and Technology, JK Lakshmipat University, Jaipur, Rajasthan, 302026, India
}

\begin{abstract}
Three distinct processes of memory have been identified. These are an encoding process, a storage process and a retrieval process. In this paper we are suggesting that this information processing theory can be understood from the application of a neurophysiological- electromagnetic theory of "brainwaves" based on known (but highly idealized) properties of synaptic action and has been formulated with implications for a large scale integration required for cognitive processes.
\end{abstract}

Keywords Memory, Brain Waves, Bloch Waves, Transmission Line, Neurophysiological-Electromagnetic Theory

\section{Introduction}

Memory of some sort is fundamental to our understanding of behaviour and mind. The ideas and theories in the study of memory have changed drastically in the last 20 years[1-3]. Earlier work took its inspiration from conditioning studies and looked at human memory in terms of associations, or connections, between stimuli and responses. Although this approach to memory resulted in the discovery of numerous important principles, it seemed to many psychologists, inadequate to account for the richness and flexibility of human memory. The current trend in the study of memory is to emphasize cognitive, or mental, processes over stimulas response associations. Three distinct processes of memory have been identified. These are an encoding process, a storage process and a retrieval process. Encoding is the process of receiving sensory input and transferring it into a form, or code, which can be stored; storage is the process of actually putting coded information in to memory; and retrieval is the process of gaining access to stored, coded information when it is needed. In the Atkinson-Shiffrin theory, memory starts with a sensory input from the environment. This input is held for a very brief time -several seconds at most- in a sensory register associated with the sensory channels (vision,, hearing, touch and so forth). Information that is attended to and recognised in the sensory may be passed on to short term memory (STM), where it is held for perhaps 20 or 30 secs. Some of the information reaching STM is processed by being rehearsed - that is by having attention focussed on it, perhaps by being repeated over and over or perhaps by being processed in some other way that

* Corresponding author:

kanadray@jklu.edu.in (Kanad Ray)

Published online at http://journal.sapub.org/ijea

Copyright (C) 2012 Scientific \& Academic Publishing. All Rights Reserved will link it up with other information already stored in memory. Information that is rehearsed may then be passed along to long term memory (LTM), information not so processed is lost. When items of information are placed in LTM, they are organised in to categories, where they may reside for days, months, years or for a lifetime. When one remembers something, a representation of the item is withdrawn or retrieved from LTM. In this paper we are suggesting that this information processing theory can be understood from the application of a neurophysiologicalelectromagnetic theory of "brainwaves" based on known (but highly idealized) properties of synaptic action and has been formulated with implications for a large scale integration required for cognitive processes[4-7]. In subsequent papers [8-9] we applied the model to suggest an explanation for the surge of electrical activity during near death experience( NDE) and coordination of complex activity for sensory information processing.

It is obvious that some physical change must take place in the nervous system when we learn something new. A theory stated by Donald Hebb (1949) may turn out to be an accurate model of the physiological process responsible for learning and memory. According to Hebb, synaptic facilitation is the biological basis of learning and memory. Individual experiences result in unique patterns of neural activity, which reverberate through neural loops. This causes structural changes in the synapses to occur. These changes make firing in the loop more likely in the future. In other words, synapses become more efficient, or facilitated. Thus for Hebb, changes in the synapses are the biological basis of memory. So when excited above a certain level, the threshold, the neuron fires, that is, it transmits an electrical signal. Synapses are one way connections. Signals pass from axon to dendrite but do not travel in the opposite direction. The signals are received mostly by the dendrites of the next cell[10-13]. 


\section{Method}

Modelling a myelinated axon as a lossy transmission line of infinite periodic structure and regarding as a cascade of identical cells(Fig.1);in previous papers[4-9] we argued that brain waves can be treated as "Bloch waves" which may provide an important mechanism for a large scale integration required between the constantly interacting areas of the brain.

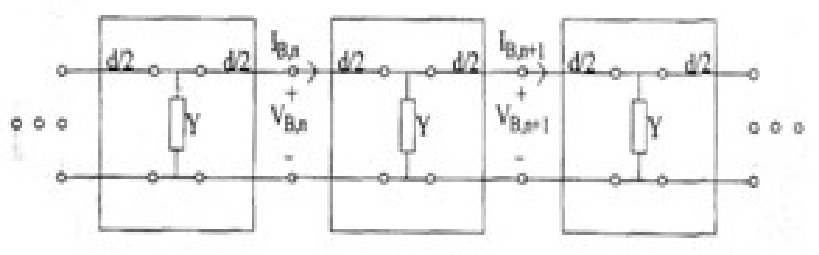

Figure 1. Transmission line axon model

Each cell is a two-port composed of a parallel admittance $\mathrm{Y}$ and two transmission line segments of length $d / 2$ at each side of the admittance. The admittance of each cell plays the role of Ranvier node, where $\mathrm{Y}=\mathrm{G}+\mathrm{j} \omega \mathrm{C}$. $\mathrm{G}$ :conductance and C:capacitance.

The Bloch impedance is different if the terminal planes of the unitary cell are shifted a distance 1 in the $\mathrm{z}$ direction $[7,8]$.

$$
Z_{B}^{\prime}=Z_{0} \frac{Z_{B}+j Z_{0} \tan \left(k_{0} l\right)}{Z_{0}+j Z_{B} \tan \left(k_{0} l\right)}
$$

Periodic structures may be analyzed in terms of the forward and backward propagating waves that can exist in each unit cell. Let the amplitudes of the forward and backward propagating waves at the nth and $(n+1)$ st terminal plane be $\mathrm{c}_{\mathrm{n}}^{+}, \mathrm{c}_{\mathrm{n}}^{-}, \mathrm{c}_{\mathrm{n}+1}^{+}$and $\mathrm{c}_{\mathrm{n}+1}^{-}[14]$

$$
\left[\begin{array}{l}
c_{n}^{+} \\
c_{n}^{-}
\end{array}\right]=\left[\begin{array}{ll}
A_{11} & A_{12} \\
A_{21} & A_{22}
\end{array}\right]\left[\begin{array}{l}
C_{n+1}^{+} \\
C_{n+1}^{-}
\end{array}\right]
$$

Solution for a Bloch wave requires:

$$
\begin{aligned}
& c_{n+1}^{+}=e^{-\gamma d} c_{n}^{+} \\
& \text {and } \quad c_{n+1}^{-}=e^{-\gamma d} c_{n}^{-} \\
& \therefore \text { eq }(1) \text { becomes } \\
& {\left[\begin{array}{rr}
A_{11}-e^{-\gamma d} & A_{12} \\
A_{21} & A_{22}-e^{\gamma d}
\end{array}\right]\left[\begin{array}{c}
c_{n+1}^{+} \\
c_{n+1}^{-}
\end{array}\right]=0}
\end{aligned}
$$

Nontrivial solution for $\mathrm{c}_{\mathrm{n}+1}{ }^{+}, \mathrm{c}_{\mathrm{n}+1}{ }^{-}$is obtained only if the determinant vanishes.

Consequently, the eigen value equation for $\gamma$ is

$$
\begin{gathered}
\mathrm{A}_{11} \mathrm{~A}_{22}-\mathrm{A}_{12} \mathrm{~A}_{21}+\mathrm{e}^{2 \gamma \mathrm{d}}-\mathrm{e}^{\gamma \mathrm{d}}\left(\mathrm{A}_{11}+\mathrm{A}_{22}\right)=0 \& \\
\cosh \gamma d=\frac{A_{11}+A_{22}}{2}
\end{gathered}
$$

since the determinant of the transmission matrix $\left(A_{11} A_{22}-A_{12} A_{21}\right)$ is 1 when normalized wave amplitudes are used.

The Bloch wave which can propagate in the periodic structure is made up from forward and backward propagating normal transmission line or wave guide waves that exist between discontinuities. When $\gamma$ has been determined from eq. (3).

The total voltage and current at the nth terminal plane will be a superposition of an incident and reflected Bloch wave.

The input impedance at the nth terminal plane is[7,8]

$$
\bar{Z}_{n}=\bar{Z} \frac{\bar{Z}_{L}+j \bar{Z} \tan (N-n) \beta d}{\bar{Z}+j \bar{Z}_{L} \tan (N-n) \beta d}
$$

One important property of this periodic structure is passband-stopband characteristics. The information contained in the eigen value equation for the propagation constant $\beta$ can be plotted on a $\mathrm{k}_{0} \beta$ (or $\omega$ - $\beta$ ) plane. The resultant plot is the Brillouin diagram[7]. The passband - stopband characteristic is the existence of frequency bands throughout which a wave propagates unattenuated along the structure separated by frequency bands throughout which the wave is cut off and does not propagate.

\section{Discussion and Conclusions}

It is believed gamma switch is a general principle of the brain, employed throughout the brain to enhance interregional communication. It is found that there are slow gamma waves and fast gamma waves coming from different brain areas, just like radio stations transmit on different frequencies. Brain filters out distracting thoughts to focus on a single bit of information[15]. The lower frequencies are used to transmit memories of past experiences, and the higher frequencies are used to convey what is happening where we are right now .The high-frequency oscillations generated by the brain are coupled to the slower theta oscillations. Stronger and more lasting memories are likely to be formed when a person is relaxed and the memory-related neurons in the brain fire in sync with certain brain waves. Synchronization in the brain is influenced by "theta waves" which are associated with relaxation but also with learning and memory formation[16]. Coupling is important because the two rhythms have different functions and operate on different spatial scales. High frequency activity is very localized while the theta rhythm is much more spatially widespread. It may be possible that brain oscillations organize neurons into cooperating groups: low-frequency waves synchronize the firing of large groups of neurons, while the higher frequencies synchronize smaller groups. Although the underlying neuronal activity is not known the oscillations may be generated spontaneously by neurons when grouped together. We are arguing that the groups of neurons modeled as transmission line behave like resonators and external stimuli within a broad spectrum of operating brain frequencies affect the firing properties of individual neurons. The nonlinear properties of an individual neuron can amplify the effects of such small fields of brain waves treated as Bloch waves. It could be possible to either have an experience, or to store or retrieve experiences in such a way as to highlight what appears important and discard the seemingly weak or unimportant factors[17]. Attention regulates the flood of sensory information pouring into the brain into a manageable stream. In particular, a lot of different areas of the brain are involved in vision. If they all competed at once, it would be chaos[18]. The predictability of memory strength is determined by spike 
timing relative to theta oscillations. These findings provide a link between the known modulation of theta oscillations by many memory-modulating behaviours and circuit mechanisms of plasticity[16]. This could explain the way we just remember certain incidents, things or dates. For other cases when information is rehearsed the transmission line resonator attains the resonant frequency and the information is stored in LTM from STM for days, months and years.

\section{REFERENCES}

[1] Morgan, C.T. et al: Introduction to psychology, Tata McGraw Hill

[2] Feldman, R. S.: Understanding psychology, Tata McGraw Hill

[3] Lahey, B. B.: Psychology :An introduction, Tata McGraw Hill

[4] Ray, K.: Modelling of the action potential propagation in nerve fibre, presented in "MP2009" in Amsterdam,1st-4th August,2009,MathPsych, 43, Society for mathematical Psychology and European Mathematical Psychology Group

[5] Ray, K.: Could brain waves be treated as Bloch waves, accepted for presentation in CNS 2010 in Montreal, Canada, 17-20th April, 2010.

[6] Ray, K.: A transmission line model of brain waves, accepted for presentation in WMVC10 at Kakatiya University, Warangal,13-15th March,2010.

[7] Ray, K. and Roy, M.K.: A theoretical basis for brain waves with implications for a large scale integration required for cognitive processes- Proceedings of the 9th IEEE International Conference on Cognitive Informatics, Beijing, China, 7-9thJuly, ICCI'10, pp.436-440, 2010, IEEE computer So- ciety

[8] Ray, K. and Roy, M.K.: A theoretical basis for surges of electroencephalogram activity and vivid mental sensation during near death experience, International Journal of Engineering Science and Technology, Vol. 2(10), 2010, pp. 5484

[9] Ray, K.: Coordinating Complex Activity: Standing and Travelling BlochWaves Play Key Role- Vol. 2(11), 2010,pp. 6691

[10] Anderson, J. A.: An introduction to neural networks, Prentice Hall India

[11] Bose, N. K. \& Liang, P.: Neural network fundamentals with graphs, algorithms and applications, Tata McGraw Hill

[12] Kartalopoulos, S. V.: Understanding neural networks and fuzzy logic, Prentice Hall India

[13] Freeman, J. A. and Skapura, R. M:Neural networks, algorithms, applications and programming techniques, Pearson Education Asia

[14] R.E. Collin, Foundations for Microwave Engg.,McGraw-Hil 1

[15] Colgin, L. L. et al.: Frequency of gamma oscillations routes flow of information in the hippocampus. Nature, 462 (7271), 2009,pp. 353

[16] Rutishauser, U.: Human memory strength is predicted by theta-frequency phase-locking of single neuron, Nature 464,2010,pp. 903

[17] Radman T,;Spike Timing Amplifies the Effect of Electric Fields on Neurons: Implications for Endogenous Field Effects The Journal of Neuroscience, March 14, 2007

[18] Buschman T. J. and Earl K. Miller.: Serial, Covert Shifts of Attention during Visual Search Are Reflected by the Frontal Eye Fields and Correlated with Population Oscillations, Neuron, 63(3),2009, pp. 386 